\title{
Non-thermal ablation of neural tissue with femtosecond laser pulses
}

\author{
F.H. Loesel ${ }^{1, *}$, J.P. Fischer ${ }^{1}$, M.H. Götz ${ }^{1}$, C. Horvath ${ }^{1,2}$, T. Juhasz ${ }^{2}$, F. Noack ${ }^{3}$, N. Suhm ${ }^{4}$, J.F. Bille ${ }^{1}$ \\ ${ }^{1}$ Department of Applied Physics, University of Heidelberg, Albert-Ueberle-Str. 3-5, 69120 Heidelberg, Germany \\ (Fax: +49-6221-549-262, E-mail: frieder.loesel@urz.uni-heidelberg.de) \\ ${ }^{2}$ Center for Ultrafast Optical Science, University of Michigan, Ann Arbor, Michigan 48109, USA \\ ${ }^{3}$ Max-Born-Institute of Nonlinear Optics and Spectroscopy, 12489 Berlin, Germany \\ ${ }^{4}$ German Cancer Research Center, 69120 Heidelberg, Germany
}

Received: 29 January 1997/Revised version: 14 July 1997

\begin{abstract}
Nonthermal in vitro ablation of bovine neural tissue by using laser-induced optical breakdown generated by ultrashort laser pulses, with durations from $100 \mathrm{fs}$ to $35 \mathrm{ps}$ and pulse energies of up to $165 \mu \mathrm{J}$, has been investigated. The experiments were performed at wavelengths ranging from 630 to $1053 \mathrm{~nm}$ by using a femtosecond Ti:Sapphire laser, a femtosecond dye laser, and a picosecond Nd:YLF laser system. Tissue ablations have been achieved by focusing the laser beam on the surface of the tissue, to a spot diameter of 5-20 $\mu \mathrm{m}$, resulting in the generation of a microplasma. Laser pulses from the Ti:Sapphire laser with $140 \mathrm{fs}$ duration showed a two times higher efficiency of ablation than the longer $30 \mathrm{ps}$ pulses from a Nd:YLF laser with an identical pulse energy. At pulse energies of $140 \mu \mathrm{J}$, single pass excisions deeper than $200 \mu \mathrm{m}$ were generated by the $140 \mathrm{fs}$ pulses. In addition, the fluence at threshold of the ablation was found to be reduced for shorter pulse durations. For 3 ps laser pulses at $630 \mathrm{~nm}$, we measured the fluence at threshold to be about $5.3 \mathrm{~J} / \mathrm{cm}^{2}$; for $100 \mathrm{fs}$ pulses from the same laser the experimental threshold was at $1.5 \mathrm{~J} / \mathrm{cm}^{2}$. Histopathological examinations and scanning electron micrographs confirm the high quality of the excisions. No sign of significant thermal damage was observed.
\end{abstract}

PACS: $42.62 . \mathrm{Be} ; 52.50 . \mathrm{Jm} ; 87.50 . \mathrm{Hj}$

The application of lasers in neurosurgery has received increasing attention in recent years, although results of early studies were not very promising [1-3]. Investigations primarily by Ascher, Beck, and Heppner discussed the advantages of using lasers in this field of medical therapy, that is, the possibility to perform slightly invasive, noncontact surgery of sensitive neural tissue (e.g., $[4,5])$. Recently, Krishnamurthy and Powers provided an extensive review on the application of lasers in neurosurgery [6].

Mainly $\mathrm{CO}_{2}$ and $\mathrm{Nd}$ :YAG lasers are used for vaporizing or coagulating the biological material. These free running or Q-switched lasers exhibit primarily a thermal interaction with

\footnotetext{
* To whom correspondence should be addressed
}

the tissue $[7,8]$. Therefore, owing to heat diffusion, thermal damage to adjacent tissue is commonly observed [8-11]. Furthermore, a big part of the irradiated tissue remains inside the brain and may lead to edema.

A completely different process of laser-tissue interaction is plasma-mediated ablation, demonstrated, e.g., by $\mathrm{Pu}-$ liafito et al. [12] and Stern et al. [13] in ophthalmologic applications. In this process, the extremely high intensity of powerful ultrashort pulses first leads to multiphoton absorption in the material. This results in the ionization of some atoms and molecules, thereby providing initial carriers ("lucky electrons") for the laser induced optical breakdown (LIOB) [12]. The free electrons and ions absorb energy from the electromagnetic field of the laser radiation by inverse bremsstrahlung, resulting in their acceleration [14]. The subsequent avalanche-like multiplication of free carriers finally leads to LIOB and the generation of a microplasma [15]. In addition, owing to the expansion of the heated plasma, a highpressure transient propagates radially from the LIOB center into the surrounding environment $[12,16]$. This shockwave also contributes to the ablation by disruption. Finally, ablation fragments are ejected out of the interaction zone [17]. Advantages of plasma-mediated ablation are the very precise cutting of tissues and the absence of undesired thermal side effects. The latter phenomenon is a result of the ultrashort interaction time: according to the works of Docchio [18] and Zysset et al. [19], a laser pulse with a duration of 30-40 ps (or shorter) generates a microplasma with a luminescence lifetime shorter than $1 \mathrm{~ns}$. Since thermal diffusion is too slow to dissipate the laser energy during this plasma lifetime [20], the thermal energy is confined to the zone of the plasma and no thermal interaction occurs in the adjacent tissue regions. Plasma-mediated ablation of brain tissue using picosecond pulses from a Nd:YLF laser system was first reported by Fischer et al. [21].

In this article, we report the experimental results on the efficiency of plasma-mediated ablation of bovine brain tissue using sub-200 fs laser pulses compared to ablation with pulses from a $30 \mathrm{ps}$ Nd:YLF laser system. We have also investigated the dependence of the threshold of plasma-mediated ablation on the duration of the laser pulses between $100 \mathrm{fs}$ 


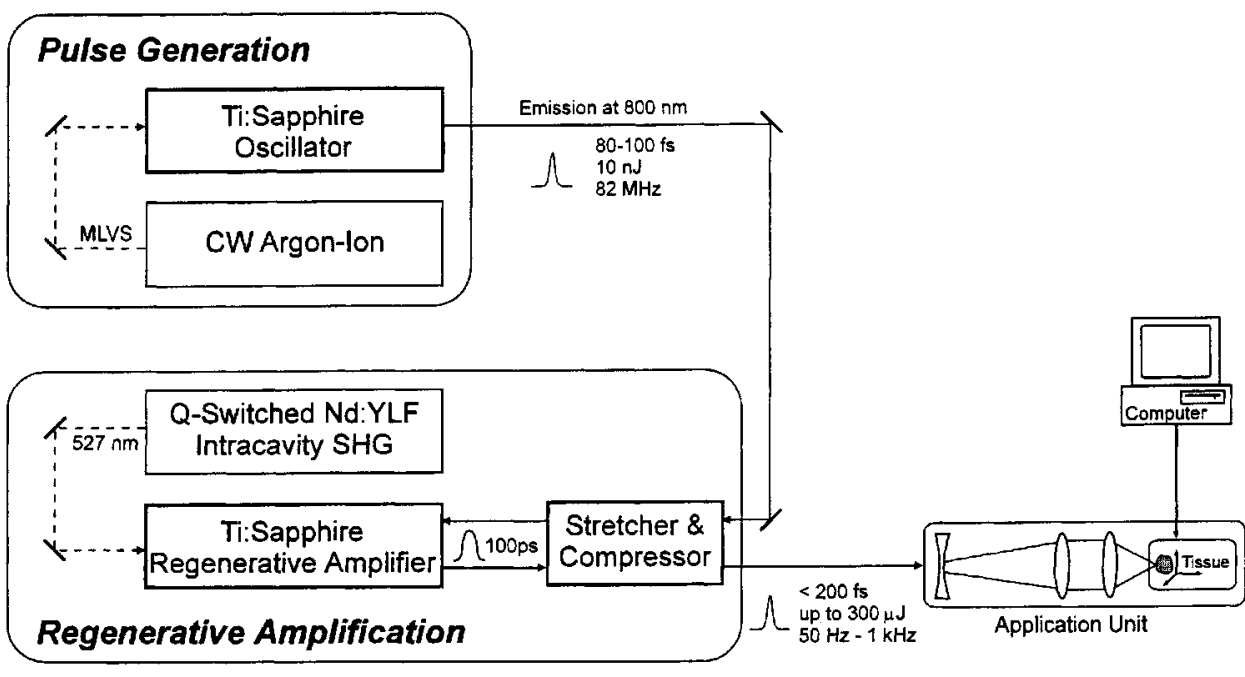

Fig. 1. Schematic of the Ti:Sapphire laser system and the application unit. Ultrashort pulses are generated in a Ti:Sapphire oscillator (top), then regeneratively amplified (bottom) by using chirped pulse amplification and directed into the computer controlled application unit (right) and $30 \mathrm{ps}$ as measured on the surface of the brain tissue. In addition, scanning electron microscopy and histopathological examinations have been performed to analyze the quality of the incisions. Our results yield information for future applications of femtosecond pulsed lasers in neurosurgery.

\section{Materials and methods}

Several laser systems, located in different laboratories, were used in this study. However, all experiments were performed by the same experimenters, to keep possible deviations in the individual experiments at a minimum.

\subsection{Laser systems}

Most of the experiments presented were performed using an ultrashort pulsed Ti:Sapphire laser system at the Max-BornInstitute of Nonlinear Optics and Spectroscopy in Berlin. The system layout is shown in Fig. 1. A Ti:Sapphire oscillator (Spectra-Physics Tsunami) is pumped with an argon-ion laser (Spectra-Physics 2080 A-12 Beamlok). Chirped pulse amplification is performed by using a stretcher/compressor unit (Quantronix Model 4820) and a Ti:Sapphire regenerative amplifier (Quantronix 4800), pumped by the frequency doubled output of a Q-switched Nd:YLF laser (Quantronix 527). Pulses with durations down to $130 \mathrm{fs}$ and energies of up to $300 \mu \mathrm{J}$ were routinely obtained at repetition rates of up to $1 \mathrm{kHz}$. The wavelength of the pulsed light was tunable in the vicinity of $800 \mathrm{~nm}$.

Owing to the tunability of the laser pulse duration, threshold experiments were performed using a multi-stage dye laser system, at the University of California at Irvine. Figure 2 gives a schematic of the dye laser system (this laser system is described in detail in [22]). Ultrashort pulses were generated in a synchronously pumped dye laser oscillator. The output of the oscillator was synchronously amplified using a two-stage dye amplifier chain pumped by the frequency doubled output of a picosecond Nd:YAG regenerative amplifier. The wavelength of the dye laser emission was set to $630 \mathrm{~nm}$ and the duration of the laser pulses was varied between $80 \mathrm{fs}$ and $3 \mathrm{ps}$. After amplification, the pulse energy was approximately $3 \mu \mathrm{J}$ at a repetition rate of $1 \mathrm{kHz}$.
For comparison, 30-35 ps pulses generated by a Nd:YLF laser system were also used to ablate neural tissue. The Nd:YLF laser was designed as a two-stage oscillator/regenerative amplifier combination to provide picosecond laser pulses at a wavelength of $1053 \mathrm{~nm}$. The system layout is shown in Fig. 3. Pulse energies of up to $1 \mathrm{~mJ}$ could be obtained at a repetition rate of $1 \mathrm{kHz}$.

\subsection{Pulse application and tissue preparation}

Fresh bovine brain tissue (white matter) was used in all our experiments. The tissue was irradiated within a few hours after extraction and was cooled until the beginning of the experiments. Saline solution was sprayed regularly on the samples to prevent the tissue surface from drying.

To determine the efficiency of plasma-mediated ablation with femtosecond laser pulses, the regeneratively amplified Ti:Sapphire laser was used in comparison to the amplified Nd:YLF laser system. The diameters of both laser beams were increased by the means of Galilean telescopes. A lens with a $50 \mathrm{~mm}$ focal length was used to focus the radiation onto the surface of the tissue samples at normal incidence. Using the knife edge method, the size of the focal spot diameter was measured to be about $20 \mu \mathrm{m}$ for both laser systems $\left(1 / e^{2} \mathrm{de}-\right.$ cay). The samples were mounted onto a motor driven $x-y-z$ translation stage. The movement of this unit was controlled by a computer system. The tissue was moved along $1.5 \mathrm{~mm}$ long lines parallel with the $x$ axis at a velocity of $0.5 \mathrm{~mm} / \mathrm{s}$. The distance between two lines was $15 \mu \mathrm{m}$. The repetition rate of the pulses was set to $100 \mathrm{~Hz}$ or $50 \mathrm{~Hz}$ in both laser systems, resulting in $5 \mu \mathrm{m}$ or $10 \mu \mathrm{m}$ spot separations, respectively. To obtain deeper excisions, the pattern ablation was repeated four or five times. When performing these multiple patterns, an adjustment of the z-position of the tissue sample along the optical axis was necessary after each scan to realign the tissue surface with the focal plane of the cutting lens. Optimum plasma spark generation was used as alignment criteria. By means of neutral density optical filters, the energy of the laser pulses was changed prior to irradiation of the tissue samples.

After irradiation the specimens were preserved in a $10 \%$ formalin solution, dehydrated in a series of graded ethanol, 


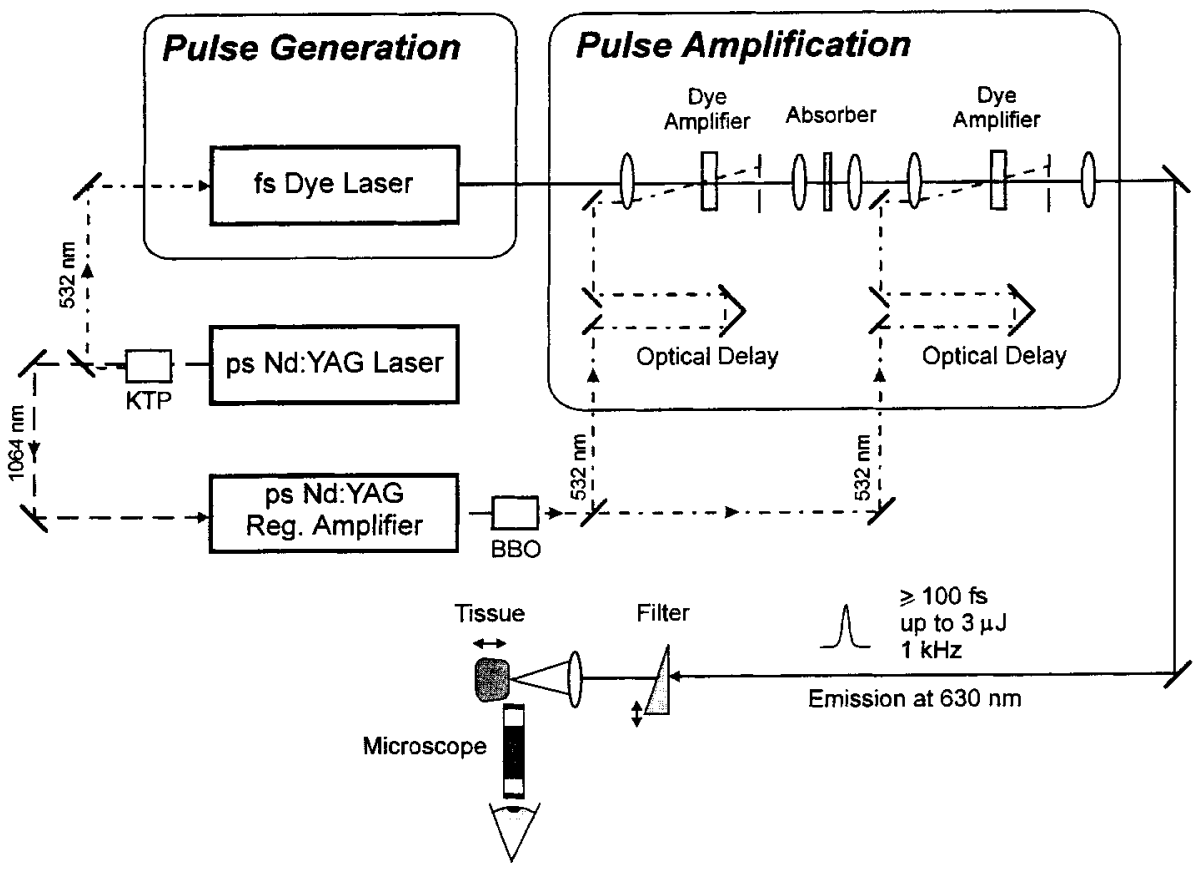

Fig. 2. The multi-stage dye laser system. A dye laser oscillator (top left) is synchronously pumped by a frequency-doubled picosecond Nd:YAG (center left). With the remaining IR output a Nd:YAG regenerative amplifier is seeded (bottom left). Its frequency doubled emission pumps the two stages of the amplifier chain for the femtosecond dye laser pulses (top right)

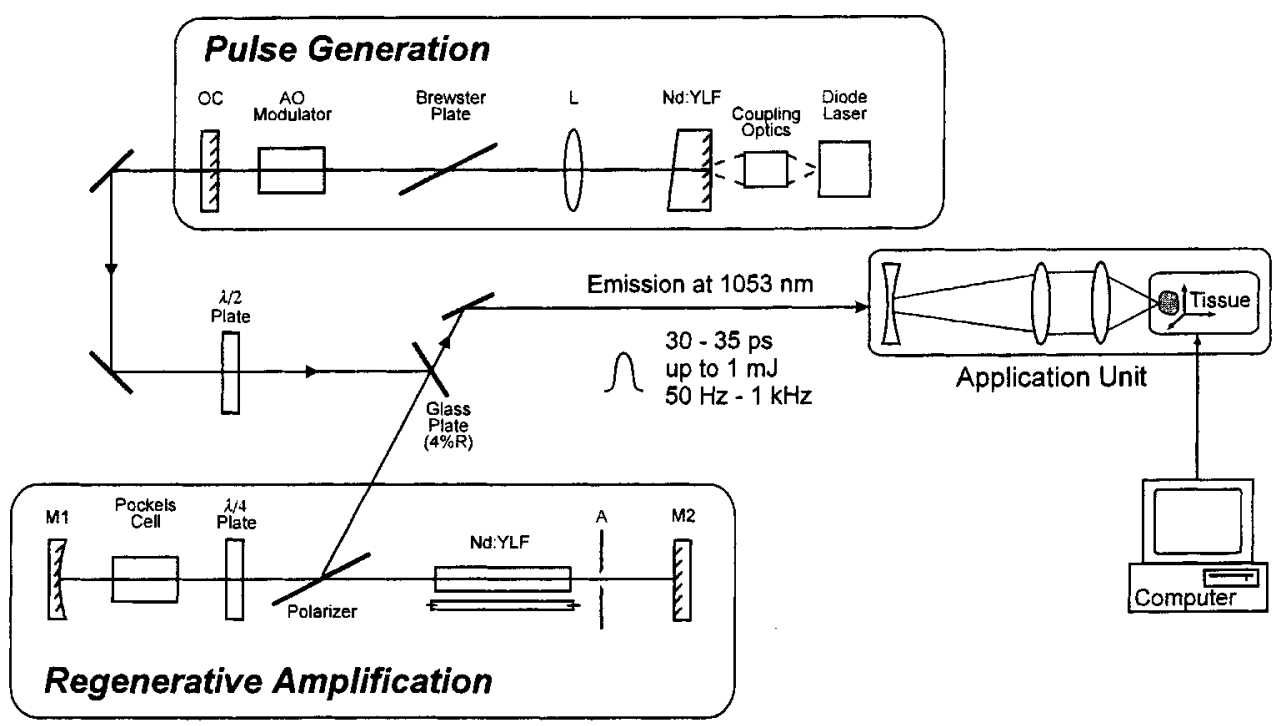

Fig. 3. Schematic of the Nd:YLF laser system. The picosecond laser pulses are generated by active modelocking in an oscillator laser (top) and then seeded into the Nd:YLF regenerative amplifier (bottom). The application unit is identical to the one used with the Ti:Sapphire laser and finally prepared for light microscopy (LM), scanning electron microscopy (SEM), or histopathological examination using LM.

To determine the damage threshold of bovine brain tissue, the beam of the dye laser system was focused onto the tissue surface at normal incidence by means of a microscope objective. Knife edge measurements yielded a spot size diameter of approximately $5 \mu \mathrm{m}$. The biological tissue was mounted on a translation stage. To ensure that the tissue surface coincided with the focal plane of the cutting lens, the sample was translated along the optical axis of the laser beam during the irradiation. In the experiment, the energy of the laser pulses was continuously decreased using a variable filter wheel. We started with above threshold pulse energies and monitored the scattered light from the unamplified output of the dye oscillator, as well as from the tissue surface by an optical microscope. Scattering of the probe beam by the ablated tissue occurred at fluences above the threshold. We defined the damage threshold as the point where the intensity of scattered light drastically decreases owing to the absence of ejected material fragments. Using the optical microscope, we observed that this event coincided with the disappearance of optical damage on the tissue surface. Starting from fluences below the threshold, we observed a drastic increase in scattered light and appearance of tissue damage at the threshold level.

\section{Results and discussion}

\subsection{Morphology of the excisions}

Figure 4 shows a typical SEM overview of a bovine brain tissue sample irradiated with laser pulses of $140 \mathrm{fs}$ in dura- 


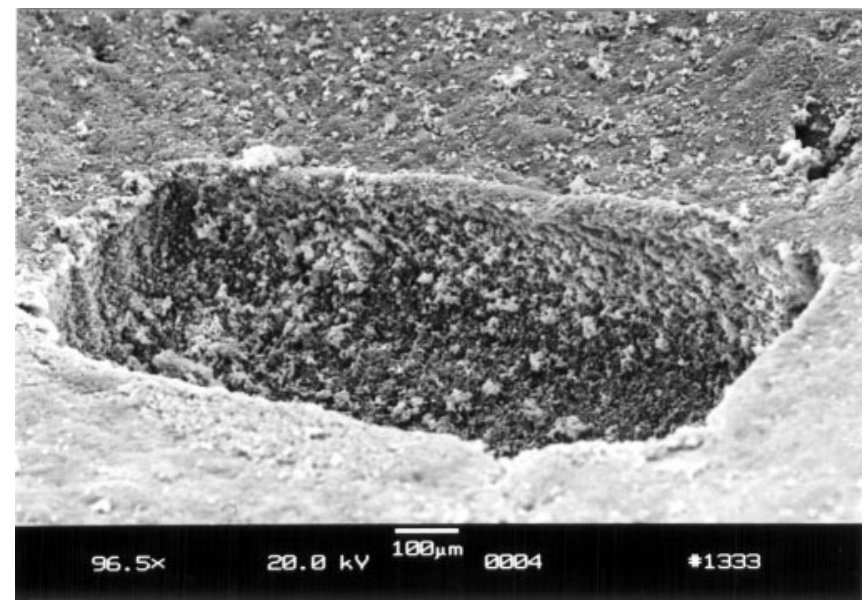

Fig. 4. SEM overview of an excision in bovine brain tissue performed by repeating a $1.5 \mathrm{~mm} \times 0.45 \mathrm{~mm}$ rectangular pattern 4 times. The tissue was moved at a velocity of $0.5 \mathrm{~mm} / \mathrm{s}$. The pulse energy was $74 \mu \mathrm{J}$ and the pulse duration was $140 \mathrm{fs}(\lambda=800 \mathrm{~nm}$, spot size: $20 \mu \mathrm{m})$. Volume of excision: $0.25 \mathrm{~mm}^{3}$. Number of applied laser pulses: 36000 . Ablated volume/pulse: $7 \times 10^{3} \mu \mathrm{m}^{3}$

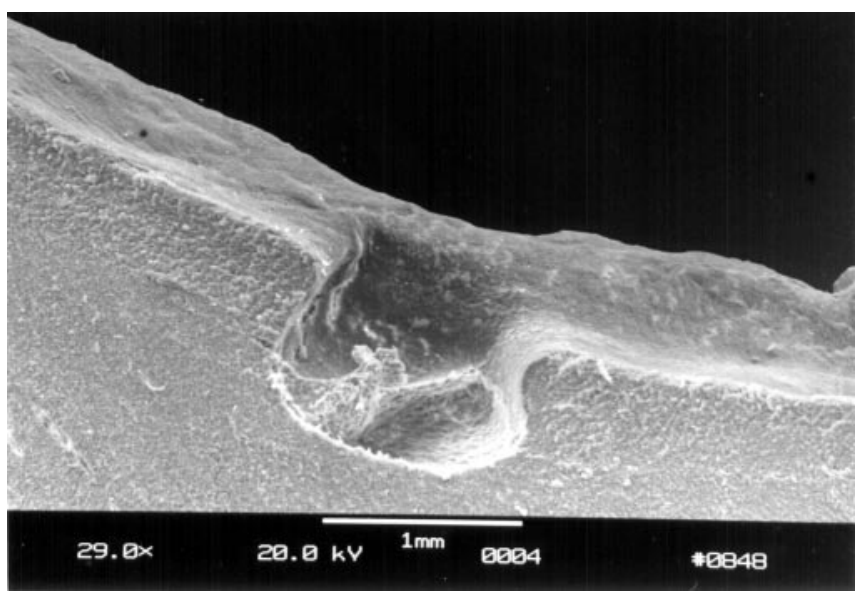

Fig. 5. SEM of a section of a bovine brain sample cut perpendicular to the tissue surface. The sample was irradiated with pulses of $165 \mu \mathrm{J}$ energy and $180 \mathrm{fs}$ duration in a rectangular pattern repeated 4 times $(\lambda=800 \mathrm{~nm}$, spot size: $20 \mu \mathrm{m})$. Volume of excision shown: $0.55 \mathrm{~mm}^{3}$. Number of applied laser pulses: 36000 . Ablated volume/pulse: $15.3 \times 10^{3} \mu \mathrm{m}^{3}$. The presence of a large piece of debris is due to the lack of tissue rinsing after irradiation

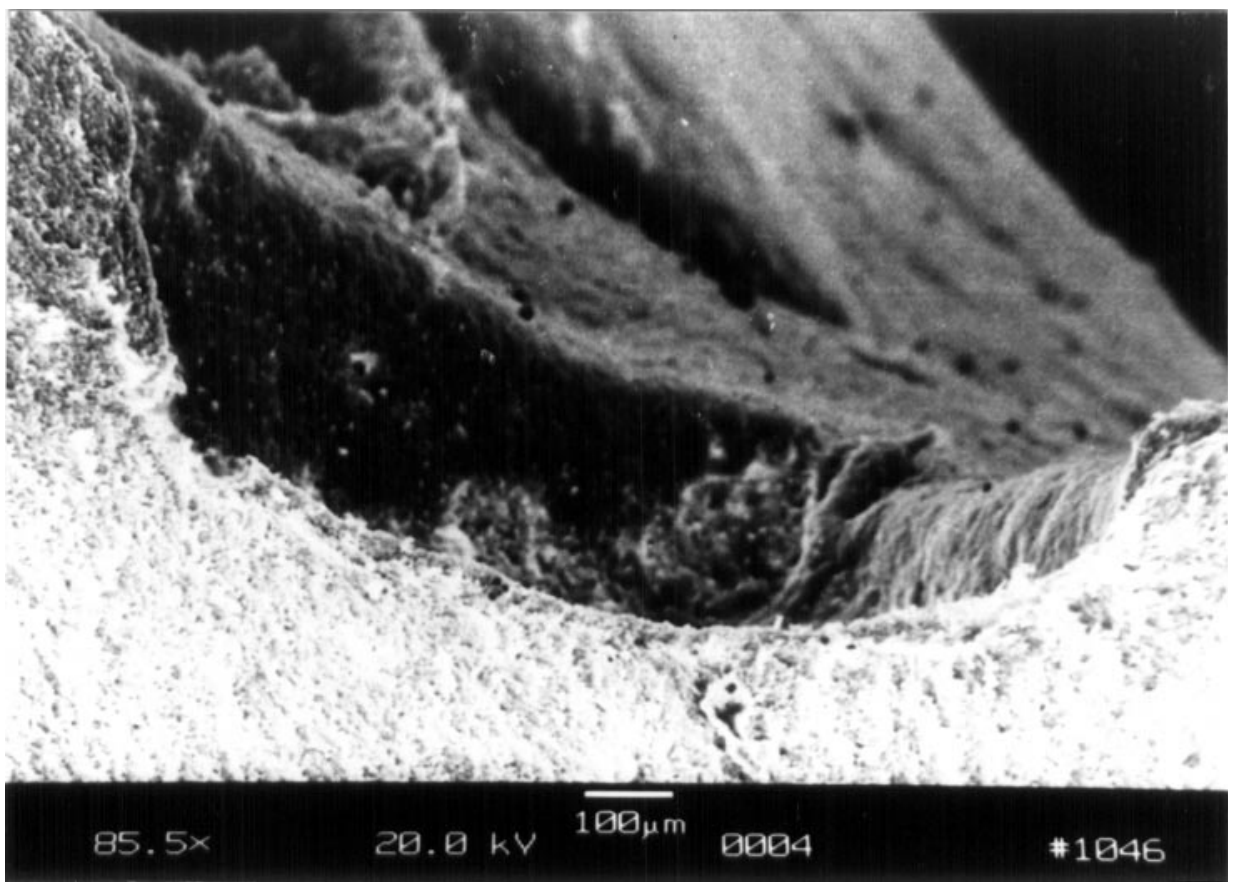

Fig. 6. SEM of a bovine brain sample section irradiated with pulses of $165 \mu \mathrm{J}$ energy and $30 \mathrm{ps}$ duration in a rectangular pattern repeated 4 times $(\lambda=1053 \mathrm{~nm}$, spot size: $20 \mu \mathrm{m})$. Sample is cut perpendicular to the tissue surface. Volume of excision shown: $0.27 \mathrm{~mm}^{3}$. Number of applied laser pulses: 36000 . Ablated volume/pulse: $7.6 \times 10^{3} \mu \mathrm{m}^{3}$ tion (FWHM) and with an energy of $74 \mu \mathrm{J}$. The rectangular pattern had the dimensions of $1.5 \times 0.45 \mathrm{~mm}$ and was repeated 4 times. The ablating laser beam generated a very welldefined deep excision in the biological material. Cutting these samples into sections parallel to the shorter side of the pattern allows further examination of the ablation edges and the depth of the excision. Figures 5 and 6 show the corresponding SEM sections of brain tissue exposed to $180 \mathrm{fs}$ pulses with $165 \mu \mathrm{J}$ from the Ti:Sapphire laser and to $30 \mathrm{ps}$ pulses from the Nd:YLF laser system. respectively. The shorter pulses generated an excision of about $800 \mu \mathrm{m}$ in depth, twice as deep as with the picosecond laser pulses (approximately $400 \mu \mathrm{m}$ ). Deeper excisions can be achieved by repeating the pattern scans more often. When ablation occurs, tissue fragments are ejected out of the damage zone [21]. Therefore, debris of ablated material can be often found covering the edges and the bottom of the excisions, as seen in Figs. 5 and 6. Figure 4 proves that most of the debris can be removed by rinsing the tissue with $\mathrm{NaCl}$ solution right after irradiation. The geometry of the excisions often appears to be slightly distorted from its original rectangular shape, as in Fig. 5. Here, the top edges apparently moved inwards, and in Fig. 6, the lower corners of the cavity appear to be smoothed. These effects are probably due to the dehydration process of the tissue prior to the SEM.

The captions of Figs. 4-6 give the total number of pulses used to generate the excisions, as well as the ablation volume per pulse. The latter was calculated by simply dividing the volume of the excision by the number of applied laser pulses. Yet, one has to consider, that owing to the spatial overlap of 
the laser pulses, data on the amount of ablated tissue are not valid for single pulse application, but they are of importance for "real world" applications.

\subsection{Efficiency of ablation}

Apart from the single excision data, we studied the dependence of the ablation rate on various parameters. Figure 7 gives quantitative results of depth measurements using single pass ablations performed at various pulse energies and repetition rates. The filled circles represent data obtained with the Ti:Sapphire laser system using pulses with durations of $140 \mathrm{fs}$ at $100 \mathrm{~Hz}$ repetition rate, and open circles indicate data from the $140 \mathrm{fs}$ pulses but at a reduced repetition rate of $50 \mathrm{~Hz}$, resulting in a smaller spatial overlap of pulses. For the sake of comparison, the filled diamonds represent data obtained with the Nd:YLF laser operating at a $30 \mathrm{ps}$ pulse duration and $100 \mathrm{~Hz}$ repetition rate. To evaluate these experiments, the irradiated samples were cut into thin slices and the depth of the excisions was measured under a light microscope by using an ocular with a micrometer scale. The measurements were averaged over at least six tissue samples for each pulse energy and then divided by the number of passes. Shown in Fig. 7 is the averaged depth of ablation for one single pass pattern as a function of pulse energy. This value is characterizing the efficiency of the ablation and is most closely related to practical applications. There is a scatter to the measured data because of the inhomogeneous nature of the samples, sample-to-sample variations, and as well as the difficult task of realigning the tissue surface after each single-pass. Therefore, error bars are included in Fig. 7. They represent the standard deviation of the mean, as calculated from the sample data. We attribute the larger errors occurring for $140 \mathrm{fs} / 100 \mathrm{~Hz}$ pulses at the two highest pulse energies to mainly two sources: first, at these two pulse energies only six samples were available for evaluation, whereas the number of samples at all other pulse energies was about 10. Second, at these high ablation depths, the geometrical distortions of the excisions appeared to be stronger after the sample preparation. That is why these depth measurements had a higher uncertainty. To some extent, beginning of autolysis may also have contributed to the errors. According to Fig. 7, the shorter femtosecond pulses exhibit a significantly higher efficiency of ablation than the picosecond pulses. The slope of the dashed line for the femtosecond pulses, in Fig. 7, is about 1.5, and for the solid line, representing the results from the picosecond pulses, is about 0.6 . Even with the repetition rate reduced by a factor of 2, thus with half of the spatial overlap, the shorter femtosecond pulses still ablate a higher amount of neural tissue than the picosecond Nd:YLF laser pulses which are at a higher repetition rate. The slope of the dotted line in Fig. 7 is about 0.7 , thereby yielding a similar slope efficiency for the $50 \mathrm{~Hz}$ femtosecond Ti:Sapphire laser pulses as for the longer picosecond pulses at $100 \mathrm{~Hz}$.

One has to be aware of the fact that the wavelengths of the femtosecond Ti:Sapphire laser $(800 \mathrm{~nm})$ and of the picosecond laser $(1053 \mathrm{~nm})$ differ by a factor of approximately 1.3. However, we believe that these slightly differing wavelengths do not contribute strongly to the phenomena of increased femtosecond ablation efficiency, since the interaction is based on a plasma effect, not on linear absorption. The

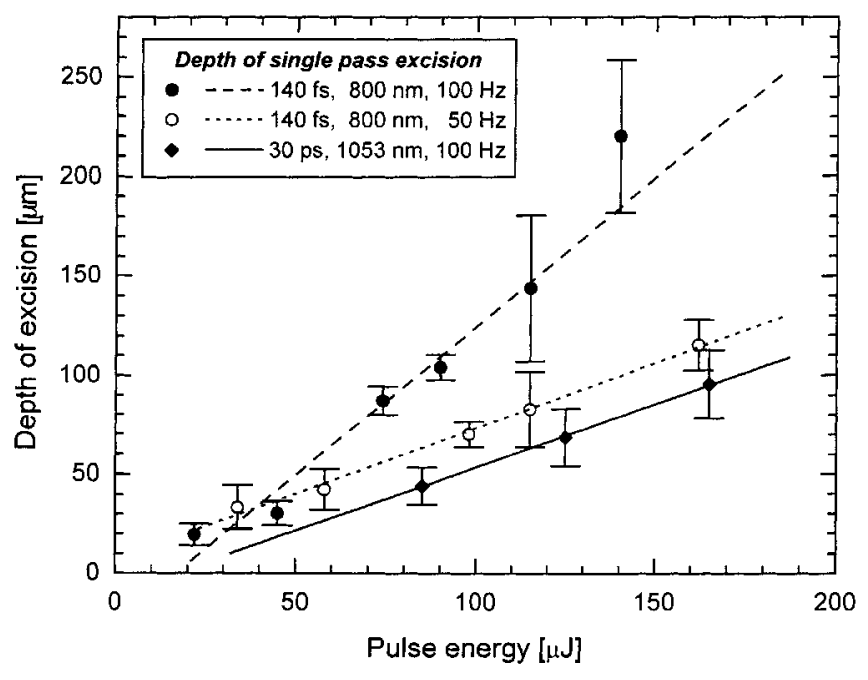

Fig. 7. The efficiency of the ablation of bovine brain tissue as measured from single pass patterns. The depth of ablation as a function of pulse energy and pulse repetition rate is displayed for two different laser systems. Filled circles: $140 \mathrm{fs}$ pulses at $100 \mathrm{~Hz}$ from the Ti:Sapphire laser emitting at $800 \mathrm{~nm}$ (dashed line is linear fit; slope $=1.5$ ). Open cirles: the same Ti:Sapphire laser at reduced repetition rate of $50 \mathrm{~Hz}$ (dotted line is linear fit; slope $=0.7$ ). Filled diamonds: 30 ps pulses at $100 \mathrm{~Hz}$ from the Nd:YLF laser emitting at $1053 \mathrm{~nm}$ (solid line is linear fit; slope $=0.6$ )

linear absorption of calf brain tissue is nearly twice as high for $1064 \mathrm{~nm}$ than, for example, for the $633 \mathrm{~nm}$ light [23], whereas, the plasma-mediated ablation exhibits a more complicated dependence on the wavelength. The initial process for LIOB with ultrashort laser pulses is the generation of "lucky electrons" by multiphoton ionization. Here, more free carriers will be generated at shorter wavelengths, because a smaller number of photons is necessary for ionization. However, for achieving macroscopic damage, the critical electron density for LIOB has to be generated by a second process, the avalanche ionization mechanism [24]. Only for extremely short pulses ( $\tau<30 \mathrm{fs}$ ), can multiphoton ionization alone provide the critical density of electrons [24]. The avalanche ionization is governed by inverse bremsstrahlung, which has a cross-section proportional to $\lambda^{2}$ [14], where $\lambda$ denotes the wavelength of the incident radiation. Consequently, in this second stage of LIOB, more free carriers are generated at longer wavelengths, in contrast to the initial multiphoton ionization. The impact of these mechanisms on the ablation rate is not yet fully understood, and we regard this study as a comparison between the efficiency of a typical picosecond and a typical femtosecond laser system.

Using the efficiency measurements, we can estimate the time needed for the ablation of a given amount of tissue with various laser parameters. Our Ti:Sapphire laser system is capable of generating $140 \mathrm{fs}$ pulses with an energy of $150 \mu \mathrm{J}$ and a repetition rate of $1 \mathrm{kHz}$. Assuming a ten times faster scanning movement than in our experiments (which could not be realized with our slow scanning system), we calculated the time needed for the ablation of $1 \mathrm{~mm}^{3}$ to be about $67 \mathrm{~s}$. The increase of the repetition rate from $100 \mathrm{~Hz}$ to $1 \mathrm{kHz}$ corresponds to an increase in the total energy delivered to the tissue in a defined time interval by one order of magnitude. Based on a ten times faster scanning speed at $1 \mathrm{kHz}$, we do not expect any changes in the quality of the ablation. The rate at which the energy is deposited is of secondary impor- 
tance [19], since the plasma luminescence governs the thermal confinement. Because of the faster scanning, the spatial separation of the single microplasmas remains unchanged. As the pulse energy also remains unchanged, we do not anticipate further mechanical damage due to shockwaves.

Today, Ti:Sapphire amplifiers with pulse energies of up to $1 \mathrm{~mJ}$ at repetition rates of $1 \mathrm{kHz}$ are available [25]. Extrapolating our measurements in Fig. 7 to this pulse energy and assuming faster scanning, we calculated an ablation time of $9 \mathrm{~s} / \mathrm{mm}^{3}$ for these laser parameters. Here, we would expect to observe stronger disruptive effects during ablation [26], since the pulse energy is increased by nearly a factor of 7 . This high energy is coupled into the acoustic transient, propagating into the surrounding tissue. Before recommending such a highenergy laser system for tissue ablation, detailed investigations should be carried out, concerning, e.g., the topic of increased plasma transmission and associated collateral damage.

\subsection{Histopathological appearance}

In order to judge the histopathological appearance of the excisions, samples were cut into $10 \mu \mathrm{m}$ thin slices parallel to the shorter side of the pattern and perpendicular to the tissue surface. Then, the slices were stained with various dyes, such as haematoxylineosin (HE), Cluever-Barrera, Kresyl violet, and Luxol fast blue. Figure 8a,b shows typical optical photomicrographs of a HE-stained brain tissue sample exposed to $115 \mu \mathrm{J}, 140 \mathrm{fs}$ laser pulses from the Ti:Sapphire laser. In this example, the pattern was repeated 4 times and the excision is about $550 \mu \mathrm{m}$ deep. The upper horizontal edges are the result of mechanical cutting with a scalpel, whereas the vertical edges and the lower horizontal edge mark the boundary of the laser excision. The sample was rinsed with saline solution after irradiation and exhibits a very clean excision without any remaining debris. Figure 8 a shows an overview over the excision at a lower magnification $(\mathrm{bar}=200 \mu \mathrm{m})$, demonstrating the absence of long range structural changes caused by the irradiation. In Fig. $8 \mathrm{~b}$ the upper right corner of the excision of Fig. 8a is magnified. The bar in Fig. 8b represents a distance of $50 \mu \mathrm{m}$. The quality of the laser-cut vertical edge is exceptionally high and comparable to the mechanical cut with a scalpel (horizontal edge). Thermal damaging (coagulation or carbonization) and structural changes in the adjacent tissue seem to be limited to a zone smaller than $5 \mu \mathrm{m}$. Overall, during our experiments no structural or thermal changes exceeding the $5 \mu \mathrm{m}$ limit were observed in the tissue adjacent to the excisions on the histopathological level, neither when using the femtosecond Ti:Sapphire laser, nor with the picosecond Nd:YLF system.

We outlined in the introduction, that in neurosurgery primarily Nd:YAG or $\mathrm{CO}_{2}$ lasers are used for tissue ablation [6]. Owing to the thermal interaction mechanism of these $\mathrm{cw}$ or Q-switched lasers, collateral damage induced by heat diffusion into the surrounding tissue is commonly observed. A chronic damage zone of at least $125 \mu \mathrm{m}$ is produced by these lasers [11]. Here, the collateral damage is separated into a smaller zone of carbonization $(20-30 \mu \mathrm{m})$ and a broader area of coagulation (about $100 \mu \mathrm{m}$ ) of the tissue. For typical power levels used in neurosurgical applications, chronic thermal damage with depths ranging from $250 \mu \mathrm{m}$ up to $1-2 \mathrm{~mm}$ is reported $[9,10]$. In general, with the $\mathrm{Nd}: \mathrm{YAG}$ or $\mathrm{CO}_{2}$ laser,
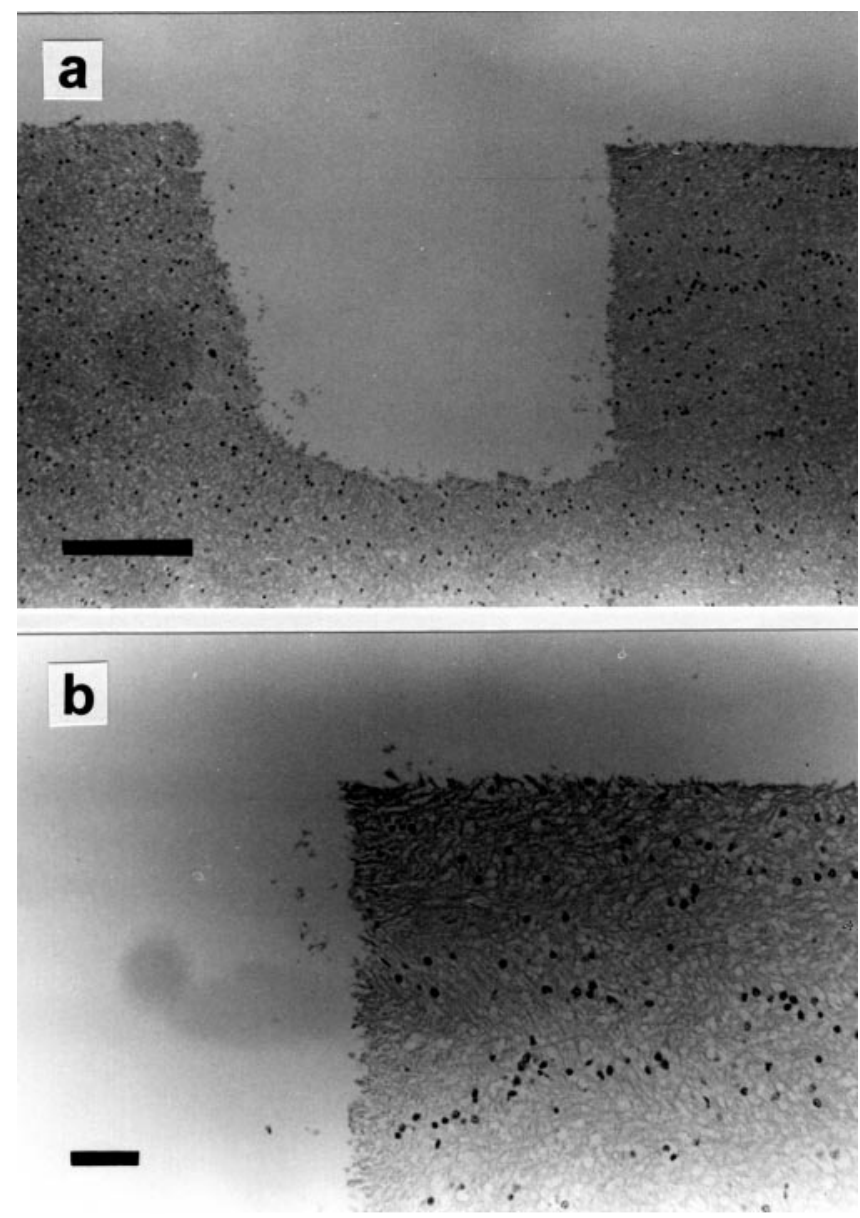

Fig. 8a,b. Optical photomicrographs showing a $550 \mu \mathrm{m}$ deep excision in bovine brain tissue generated by using $115 \mu \mathrm{J}$ Ti:Sapphire laser pulses of $140 \mathrm{fs}$ duration with a rectangular pattern repeated 4 times $(\lambda=800 \mathrm{~nm}$, spot size: $20 \mu \mathrm{m}$ ). The sample was stained with HE. Volume of excision: $0.37 \mathrm{~mm}^{3}$. Number of applied laser pulses: 36000 . Ablated volume/pulse:

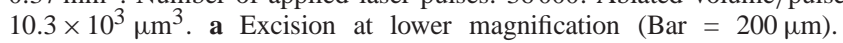
b Upper right edge of excision in more detail $(\mathrm{Bar}=50 \mu \mathrm{m})$

the observed collateral damage is about two orders of magnitude larger than with the femtosecond laser system, where the heat is confined to the zone of the microplasma.

\subsection{Threshold measurements}

Results of threshold measurements for the plasma-mediated ablation of bovine brain tissue are given in Fig. 9. The diagram shows the laser fluence at the threshold of ablation as a function of pulse duration. Both axes have a logarithmic scaling. The fluence at the threshold is calculated using the measured values for the pulse energy and the focal spot size. In addition to the data obtained with the dye laser system, which are marked by filled squares, the threshold fluence measured with $35 \mathrm{ps} \mathrm{Nd:YLF} \mathrm{laser} \mathrm{pulses} \mathrm{[21]} \mathrm{is} \mathrm{shown} \mathrm{as} \mathrm{an}$ open diamond. The plotted data points are mean values of the threshold levels, averaged over 3-5 tissue samples. The error bars are calculated from the uncertainty in the measurement of the focal spot sizes. Compared to these errors, the statistical uncertainty of the threshold measurements can be neglected. It is observed that the fluence threshold is decreasing with 


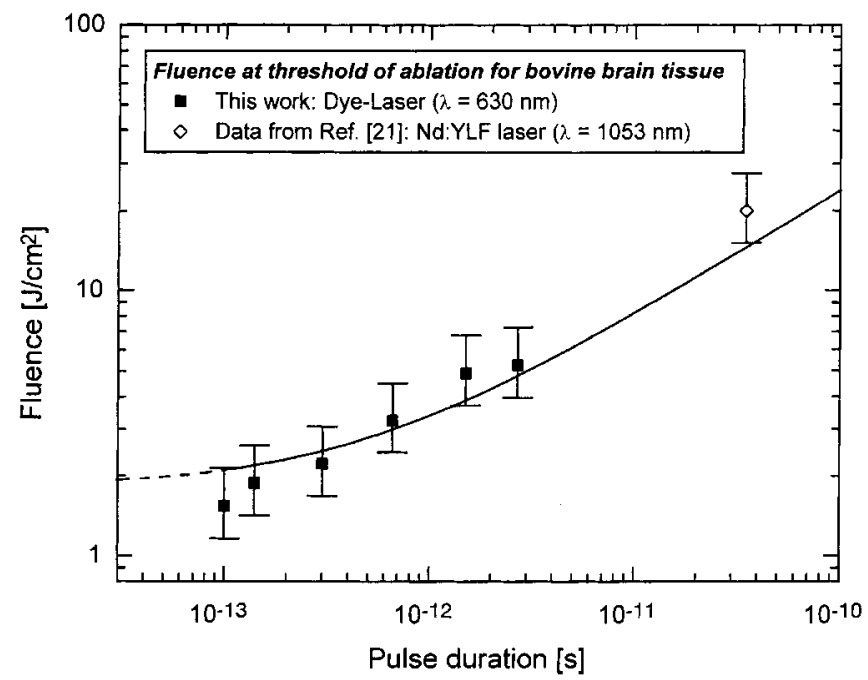

Fig. 9. Laser fluence at the threshold of ablation of bovine brain tissue as a function of the pulse duration. Filled squares: data obtained with pulses from the dye laser system at $630 \mathrm{~nm}$. Open diamond: data from [21], obtained with pulses from the Nd:YLF laser system. The solid line represents the fit from a model describing the dependence of the LIOB threshold fluence on the pulse duration (see text)

shorter pulse durations. With the Nd:YLF laser at pulse durations of $35 \mathrm{ps}$, a fluence of about $20 \mathrm{~J} / \mathrm{cm}^{2}$ is needed for ablation [21], whereas with the dye laser operating at pulse widths of $100 \mathrm{fs}$, a fluence of about $1.5 \mathrm{~J} / \mathrm{cm}^{2}$ is sufficient for ablating the bovine neural tissue. Shown as a solid line in Fig. 9 is a fit from a theoretical model for the dependence of the LIOB threshold fluence on the pulse duration [27]. This model describes the temporal behavior of the free electron density by analyzing the corresponding generation and loss mechanisms. According to the model, summarized in [27], the threshold fluence $E_{\mathrm{th}}$ of LIOB is related to the duration of the laser pulse $\tau$ as

$\eta E_{\mathrm{th}} \cong 9+\sqrt{40.5+\frac{\tau}{2 \tau_{\mathrm{c}}}}+\frac{\tau}{\tau_{\mathrm{d}}}$,

where $\tau_{\mathrm{c}}$ and $\tau_{\mathrm{d}}$ are the time constants for inelastic collision and for diffusion of the free electrons, respectively, and $\eta$ is the ionization probability of the material. Assuming $\tau_{\mathrm{c}}=1 \mathrm{fs}$ and $\tau_{\mathrm{d}}=500 \mathrm{ps}$ [27] and using our measured values for $E_{\mathrm{th}}$, we calculated the ionization probability for bovine brain tissue and found $\eta=9.8 \mathrm{~cm}^{2} / \mathrm{J}$ as the mean value. The solid line in Fig. 9 represents the prediction of the dependence of LIOB threshold fluence on the pulse duration for bovine brain tissue by using our measured mean $\eta$. Our experimental data are in good agreement with the theoretical model. At pulse durations of the order of several picoseconds, we found a square root dependence of the threshold fluence on the pulse duration. At shorter pulse durations a weaker dependence was observed. Deviations from the model at pulse durations of $100 \mathrm{fs}$ and shorter might have their origin in the fact that we do not account for multiphoton phenomena. We outlined in the previous sections that the avalanche ionization is the main mechanism for macroscopic tissue damage. Nevertheless, for pulse durations below $100 \mathrm{fs}$, multiphoton ionization might be the dominating mechanism. Ultimately, for laser pulses shorter than $30 \mathrm{fs}$, LIOB should be purely governed by multiphoton ionization, according to Stuart et al. [24]. Also, recent experimental findings suggest the increasing significance of multiphoton ionization for optical damage in the time domain below $100 \mathrm{fs}$ [28]. As our experimental threshold data are in good agreement with our theoretical model down to pulse durations of approximately $150 \mathrm{fs}$, we conclude that the multiphoton phenomena do not have a major impact on macroscopic tissue damage in the time domain $>150 \mathrm{fs}$.

It is important to point out that the $35 \mathrm{ps}$ data point represents results obtainable from a typical picosecond laser system, as compared to results obtainable from a typical femtosecond laser system. In case of the threshold studies, the wavelengths of the Nd:YLF laser $(1053 \mathrm{~nm})$ and the dye laser $(630 \mathrm{~nm})$ differed by a factor of nearly 1.7 . Considering the single mechanisms during LIOB, we expect some influence of this substantial difference in wavelength on the threshold of LIOB. According to Stuart et al. [24], the threshold fluence of $35 \mathrm{ps}$ pulses at a wavelength of $630 \mathrm{~nm}$ should be slightly lower than at $1053 \mathrm{~nm}$, in correspondence with the prediction of our model. Nevertheless, the data from $100 \mathrm{fs}$ to $3 \mathrm{ps}$ are obtained with a single laser system and clearly demonstrate the decrease of LIOB threshold fluence for neural tissue at shorter pulse durations.

The higher efficiency of the femtosecond Ti:Sapphire laser pulses compared to the picosecond Nd:YLF laser pulses may be partly explained by the lower threshold for the shorter pulses. With shorter pulses laser induced breakdown occurs already at larger laser beam diameters before the focal spot is reached. Therefore, the volume of the plasma is expected to be larger. This may result in larger volumes of material being ablated by a single pulse, thus leading to the high ablation efficiency.

\subsection{Applications}

There are several applications for precise small volume ablations inside the human brain. These include, for example, functional neurosurgery such as thalamotomy and the treatment of hydrocephalus. In these neurosurgical applications, tissue with volumes of up to $4 \mathrm{~mm}^{3}$, typically, has to be removed. According to the calculations presented in the previous sections, this amount of tissue could be ablated in under $5 \mathrm{~min}$ by using our Ti:Sapphire laser system at $1 \mathrm{kHz}$ repetition rate and $150 \mu \mathrm{J}$ pulse energy. This time is short enough for being of clinical relevance. For these laser parameters, the ablating beam should be scanned ten times faster over the tissue surface than in this experiment. This would avoid a higher spatial overlap of subsequent pulses and therefore would avoid a significant change of the efficiency. From a technical point of view, such scanning speeds can be easily realized. The resection of larger tissue volumes, e.g., small tumors, could be performed at higher pulse energies still within reasonable times.

Owing to the problems of significant pulse stretching and fiber damage, delivery of the femtosecond laser pulses by fiber systems is not practicable. Therefore, a novel laser probe was developed for minimal invasive laser neurosurgery [29]. The prototype probe has a diameter of only $5.5 \mathrm{~mm}$ and may be inserted into the skull to a depth of $12 \mathrm{~cm}$. Beam steering 
and delivery of the probe is performed by mirrors and micro optics. No fibers are needed in the setup. By using this laser probe, we should be able to reach even deep-seated regions and tumors for treatment.

\section{Conclusion}

Our in vitro experiments indicate that femtosecond pulsed lasers are suitable for applications in neurosurgery and have several advantages compared to the classical neurosurgical laser systems. Using femtosecond laser pulses, neural tissue can be removed without any significant sign of thermal or structural damage to adjacent tissue. In addition, the threshold fluence needed for initiating the ablation process is lower for the shorter femtosecond pulses than for picosecond or nanosecond laser pulses. Therefore, lower pulse energies can be used for the removal of neural tissue if femtosecond laser pulses are applied. This results in reduced shockwave effects from the pressure transient, since the spatial extent of the shockwave is very small when using low energy femtosecond pulses, as compared to the shockwave from picosecond or nanosecond plasma-mediated ablation [26]. In addition, in comparison to longer picosecond laser pulses from a Nd:YLF laser, the ablation with the Ti:Sapphire femtosecond pulses was found to be far more efficient.

Functional neurosurgery, specially, could benefit from the highly localized and yet efficient ablation process achieved with the femtosecond lasers. The pulses could be delivered to the desired neural region by means of a novel stereotactic probe without increasing the pulse width of the ultrashort pulses due to dispersion introduced by fibers. By using this probe, first in vivo experiments could yield further insight into the possible functional collateral damage of ultrashort laser pulses to living tissue.

In conclusion, we demonstrated that femtosecond laser systems could be promising tools for a variety of applications in precise nonthermal removal of neural tissue, which could be performed using minimally invasive techniques.

Acknowledgements. The authors wish to thank Prof. Dr. D. Komitowsky at the DKFZ Heidelberg for the histopathological examinations and B. Schagen and Dr. T. Pioch for their assistance with the SEM. They are also indebted to Prof. W.E. Bron at UC Irvine for providing the femtosecond dye laser system. F.H. Loesel is especially grateful to the DFG (LO 629/1-1) for their support.

\section{References}

1. L. Liss, R. Roppel: Neurology 16, 783 (1966)

2. H.L. Rosomoff, F. Carroll: Arch. Neurol. 14, 143 (1966)

3. S. Stellar, T.A. Polanyi, H.C. Bredemeier: Med. Biol. Eng. 8, 549 (1970)

4. O.J. Beck: Neurosurg. Rev. 3, 261 (1980)

5. P.W. Ascher, F. Heppner: Neurosurg. Rev. 7, 123 (1984)

6. S. Krishnamurthy, S.K. Powers: Lasers Surg. Med. 15, 126 (1994), and references therein

7. J.L. Boulnois: Lasers Med. Sci. 1, 47 (1986)

8. M.H. Niemz: Laser-tissue interactions (Springer, Heidelberg 1996)

9. O.J. Beck, J. Wilske, J.L. Schönberger, W. Gorisch: Neurosurg. Rev. 1, 31 (1979)

10. R.E. Wharen Jr., R.E. Anderson, B. Scheithauer, T.M. Sundt: J. Neurosurg. 60, 531 (1984)

11. G.F. Walter, P.W. Ascher, E. Ingolitsch: J. Neurol. Neurosurg. Psychiatry 47, 745 (1984)

12. C.A. Puliafito, R.F. Steinert: IEEE J. Quantum Electron. 20, 1442 (1984), and references therein

13. D. Stern, R.W. Schoenlein, C.A. Puliafito, E.T. Dobi, R. Birngruber, J.G. Fujimoto: Arch. Ophthalmol. 107, 587 (1989)

14. J.F. Ready: Effects of high-power laser radiation, (Academic Press, New York 1971)

15. N. Bloembergen: IEEE J. Quantum Electron. 10, 375 (1974)

16. C.E. Bell, J.A. Landt: Appl. Phys. Lett. 10, 46 (1967)

17. J.P. Fischer, T. Juhasz, J.F. Bille: Appl. Phys. A 64, 181 (1997)

18. F. Docchio: Europhys. Lett. 6, 407 (1988)

19. B. Zysset, J.G. Fujimoto, T.F. Deutsch: Appl. Phys. B 48, 139 (1989)

20. S.L. Jaques: Appl. Opt. 32, 2447 (1993)

21. J.P. Fischer, J. Dams, M.H. Götz, E. Kerker, F.H. Loesel, C.J. Messer, M.H. Niemz, N. Suhm, J.F. Bille: Appl. Phys. B 58, 493 (1994)

22. T. Juhasz, G.O. Smith, S.M. Metha, K. Harris, W.E. Bron: IEEE J. Quantum Electron. 25, 1704 (1989)

23. W.Cheong, S.A.Prahl, A.J. Welch: IEEE J. Quantum Electron. 26, 2166 (1990)

24. B.C. Stuart, M.D. Feit, A.M. Rubenchik, B.W. Shore, M.D. Perry: Phys. Rev. Lett. 74, 2248 (1995)

25. 5. Backus, J. Peatross, C.P. Huang, M.M. Murnane, H.C. Kapteyn: Opt. Lett. 20, 2000 (1995)

26. T. Juhasz, G. Kastis, C. Suarez, Z. Bor, W.E. Bron: Lasers Surg. Med. 19, 23 (1996)

27. F.H. Loesel, M.H. Niemz, J.F. Bille, T. Juhasz: IEEE J. Quantum Electron. 32, 1717 (1996)

28. W. Kautek, J. Krüger, M. Lenzner, S. Sartania, C. Spielmann, F. Krausz: Appl. Phys. Lett. 69, 3146 (1996)

29. J. Wahrburg, K.-U. Schmidt, M.H. Götz, K. Kappings, S. Gölz: Mechatronics 6, 479 (1996) 